\title{
Neuronal Nogo-A upregulation does not contribute to ER stress-associated apoptosis but participates in the regenerative response in the axotomized adult retina
}

\author{
V Pernet ${ }^{\star, 1}$, S Joly ${ }^{2}$, D Dalkara ${ }^{3}$, O Schwarz ${ }^{1}$, F Christ ${ }^{1}$, D Schaffer ${ }^{3}$, JG Flannery ${ }^{4}$ and ME Schwab ${ }^{1}$
}

Nogo-A, an axonal growth inhibitory protein known to be mostly present in CNS myelin, was upregulated in retinal ganglion cells (RGCs) after optic nerve injury in adult mice. Nogo-A increased concomitantly with the endoplasmic reticulum stress (ER stress) marker C/EBP homologous protein (CHOP), but CHOP immunostaining and the apoptosis marker annexin V did not co-localize with Nogo-A in individual RGC cell bodies, suggesting that injury-induced Nogo-A upregulation is not involved in axotomyinduced cell death. Silencing Nogo-A with an adeno-associated virus serotype 2 containing a short hairpin RNA (AAV2.shRNANogo-A) or Nogo-A gene ablation in knock-out (KO) animals had little effect on the lesion-induced cell stress or death. On the other hand, Nogo-A overexpression mediated by AAV2.Nogo-A exacerbated RGC cell death after injury. Strikingly, however, injury-induced sprouting of the cut axons and the expression of growth-associated molecules were markedly reduced by AAV2.shRNA-Nogo-A. The axonal growth in the optic nerve activated by the intraocular injection of the inflammatory molecule Pam3Cys tended to be lower in Nogo-A KO mice than in WT mice. Nogo-A overexpression in RGCs in vivo or in the neuronal cell line F11 in vitro promoted regeneration, demonstrating a positive, cell-autonomous role for neuronal Nogo-A in the modulation of axonal regeneration.

Cell Death and Differentiation (2012) 19, 1096-1108; doi:10.1038/cdd.2011.191; published online 23 December 2011

The membrane protein Nogo-A is one of the best characterized myelin-derived inhibitors for neurite outgrowth. ${ }^{1}$ The blockade of Nogo-A or its receptor or the systemic deletion of Nogo-A in knock-out mice (KO) enhanced axonal plasticity in the injured spinal cord and improved motor function recovery. ${ }^{2,3}$ After optic nerve crush, the blockade of Nogo-A or cognate molecules enabled retinal ganglion cell (RGC) axons to regrow, but only to a limited extent. ${ }^{4-6}$ The stimulation of the neuronal growth program by inflammatory molecules such as the toll-like receptor 2 agonists zymosan or Pam3Cys had a somewhat stronger effect on optic axon regeneration. ${ }^{7-9}$ The inhibition of the Nogo-A receptor NgR1 or its down-stream mediator Rho-A exerted synergistic effects on optic axon regrowth when combined with inflammatory molecules. ${ }^{4,10}$

However, recent studies also suggested that the intrinsic growth capacity of the injured retinal neurons as well as their responsiveness to external growth factor stimulation are impaired after axonal injury; after optic nerve lesion, increased levels of phosphatase and tensin homolog (PTEN) and tuberous sclerosis complex 1 (TSC1) inhibited the mammalian target of rapamycin (mTOR)-dependent protein synthesis. ${ }^{11}$ In addition, the responsiveness of RGCs to Ciliary Neurotrophic Factor (CNTF) was reported to be compromised by the intracellular upregulation of suppressor of cytokine signaling 3 (SOCS3), a negative regulator of the Janus Kinase 3/Signal Transducer and Activator of Transcription 3 (Jak3/Stat3) pathway. ${ }^{12}$

Although Nogo-A occurs mostly in oligodendrocytes in the adult CNS, subtypes of neurons also express the protein, but its function in these cells is unknown. Here, we found that the neuronal content of Nogo-A was increased in RGC neurons after optic nerve injury, similar to results recently described for cortical and thalamic neurons after stroke. ${ }^{13,14}$ This opens the possibility that neuronal Nogo-A may have a role in the cell

${ }^{1}$ Brain Research Institute, University of Zürich, and Department of Biology ETH Zürich, Zürich, Switzerland; ${ }^{2}$ Laboratory for Retinal Cell Biology, Department of Ophthalmology, University of Zurich, Zurich, Switzerland; ${ }^{3}$ Department of Chemical Engineering, Departmant of Bioengineering, and Helen Wills Neuroscience Institute, University of California at Berkeley, Berkeley, CA, USA and ${ }^{4}$ Department of Molecular and Cellular Biology and Helen Wills Neuroscience Institute, University of California at Berkeley, Berkeley, CA, USA

*Corresponding author: V Pernet, Brain Research Institute, University of Zürich, and Department of Biology ETH Zürich, Winterthurerstrasse, 190, Room 55J34a, Zürich, Switzerland, CH-8057. Tel: + 414463 53256; Fax: + 414463533 03; E-mail: vincent.pernet@yahoo.ca; pernet@hifo.uzh.ch

Keywords: neuronal Nogo-A; optic nerve injury; retinal ganglion cells; axonal regeneration; ER stress

Abbreviations: AAV, adeno-associated virus; ATF, activating transcription factor; BDNF, brain-derived neurotrophic factor; Bip/GRP78, 78 kDa glucose-regulated protein; CHOP/GADD153, C/EBP homologous protein/growth arrest- and DNA damage-inducible gene 153; CNTF, ciliary neurotrophic factor; CTb-594, cholera toxin $\beta$ subunit-alexa594; DRG, dorsal root ganglion; elF2 $\alpha$, eukaryotic translation initiation factor $2 \alpha$; FGF2, fibroblast growth factor 2; FL, fiber layer; GAP-43, growthassociated protein 43; GAPDH, glyceraldehyde 3-phosphate dehydrogenase; GCL, ganglion cell layer; GFAP, glial fibrillary acidic protein; GFP, green fluorescent protein; GS, glutamine synthetase; ILM, inner limiting membrane; INL, inner nuclear layer; IPL, inner plexiform layer; Jak3, janus kinase 3; LIF, leukemia inhibitory factor; LINGO1, leucine rich repeat and Ig domain containing 1; MAG, myelin-associated glycoprotein; mTOR, mammalian target of rapamycin; NgR1, Nogo66 receptor 1; OLM, outer limiting membrane; ONL, outer nuclear layer; OPL, outer plexiform layer; OS, outer segment; Pam3Cys, (S)-(2,3-bis(palmitoyloxy)-(2RS)-propyl)-Npalmitoyl-(R)-Cys-(S)-Ser(S)-Lys(4)-OH^trihydrochloride; PTEN, phosphatase and tensin homolog; qRT-PCR, quantitative real-time polymerase chain reaction; RGCs, retinal ganglion cells; RTN, reticulon; shRNA, small hairpin ribonucleic acid; SOCS3, suppressor of cytokine signaling 3; Sprr1A, small proline-rich protein 1A; Stat3, signal transducer and activator of transcription 3; TNF- $\alpha$, tumor necrosis factor $\alpha$; TSC1, tuberous sclerosis complex 1

Received 04.7.11; revised 28.10.11; accepted 17.11.11; Edited by M Deshmukh; published online 23.12.11 
death/survival and/or regeneration response of injured CNS neurons. ${ }^{14}$ Interestingly, the genetic deletion of Nogo-A/B in mutant mice worsened the motor and cognitive deficits after traumatic brain injury and accelerated the degeneration of motorneuron axons in a model of amyotrophic lateral sclerosis (ALS). ${ }^{14-16}$ A neuroprotective effect of Nogo was proposed to be related to an attenuation of endoplasmic reticulum (ER) stress. ${ }^{15}$ Only few and contradictory observations are available on such a role of Nogo (reticulon 4 (RTN4)) or other RTN proteins, and they rely mostly on in vitro or overexpression experiments. ${ }^{15,17-21}$ We therefore investigated axonal regeneration and survival of RGCs after optic nerve crush in mice with systemic Nogo-A deletion (KO) or neuron-specific knock down using adeno-associated virus vector of serotype 2 (AAV2) that selectively infect RGCs in the retina. For the first time, our work demonstrates that the exogenous increase of neuronal Nogo-A driven by AAV2.Nogo-A, but not the endogenous upregulation of neuronal Nogo-A because of axonal damage enhanced RGC cell loss. Our results also reveal a positive function for neuronal Nogo-A on the intrinsic growth properties of damaged neurons.

\section{Results}

Nogo-A is specifically upregulated in RGCs after axotomy. In the intact retina of adult mice Nogo-A was detected by immunofluorescence almost exclusively in Müller cells; in freshly isolated Müller cells the protein was localized in the inner processes of the Müller glia (end-feet) (Figures $1 \mathrm{a}$ and $\mathrm{b}$ ). The protein Nogo-B, a small splice form of Nogo-A, was similarly concentrated in Müller cell extensions (data not shown). After axotomy, Nogo-A remained unchanged in the glial end-feet, whereas the gliosis marker Glial Fibrillary Acidic Protein (GFAP) was strongly upregulated and spread apically in the radial processes of the Müller cells (Figures 1c and $d$ ). The
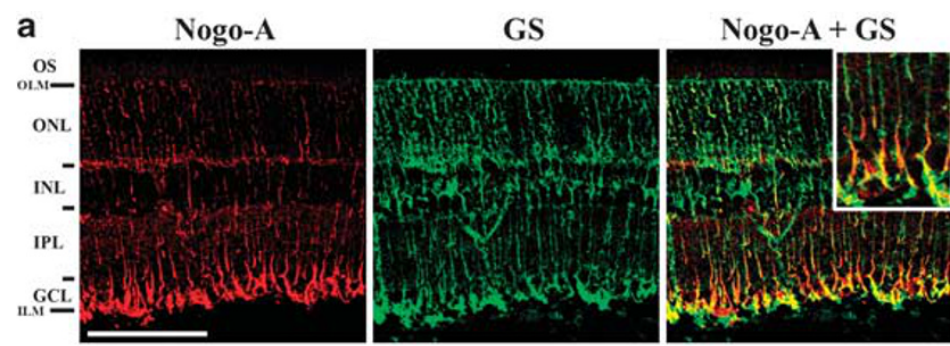

b

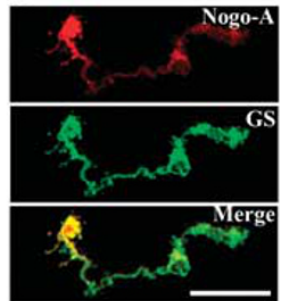

C

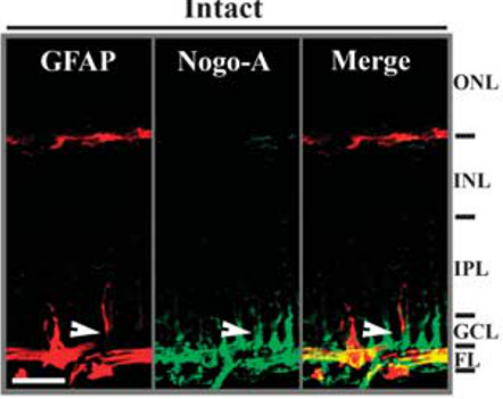

d



e

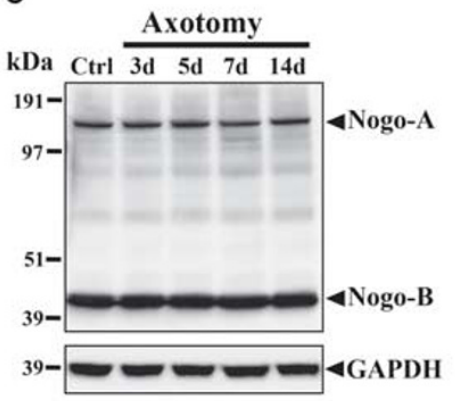

f

Vimentin

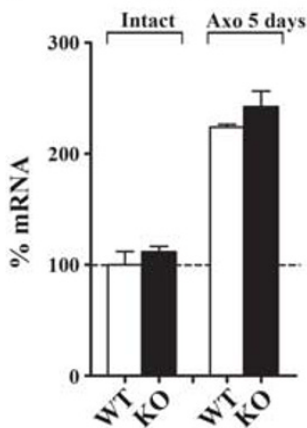

g

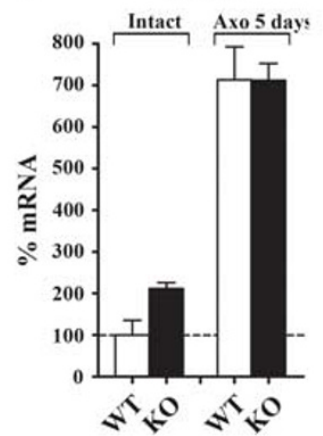

Figure 1 Expression of Nogo-A in the Müller glia of the intact and injured retina. The protein distribution of Nogo-A was analyzed by immunofluorescence on retinal sections and retinal flat-mounts of unoperated and injured retinae after optic nerve cut. (a) On slices of intact retinae, Nogo-A co-localized with GS, a specific marker for Müller glia, in the inner side of the radial processes called end-feet. (b) In vitro, freshly dissociated Müller cells presented the same accumulation of Nogo-A in the end-feet, whereas GS was evenly distributed throughout the cytoplasmic space. (c and d) Seven days after optic nerve axotomy, GFAP spread apically in Müller cell extensions (arrowheads), whereas Nogo-A protein remained limited in the end-feet. (e) The western blot analysis failed to show a change in Nogo-A and Nogo-B protein expressions at different time points after optic nerve cut. ( $\mathbf{f}$ and $\mathbf{g}$ ) Five days after injury, the mRNA increase of the gliosis markers vimentin and GFAP was similar between Nogo-A KO and WT lysates. Scale bars: $\mathrm{A}=100 \mu \mathrm{m}, \mathrm{B}, \mathrm{C}=25 \mu \mathrm{m}$ 

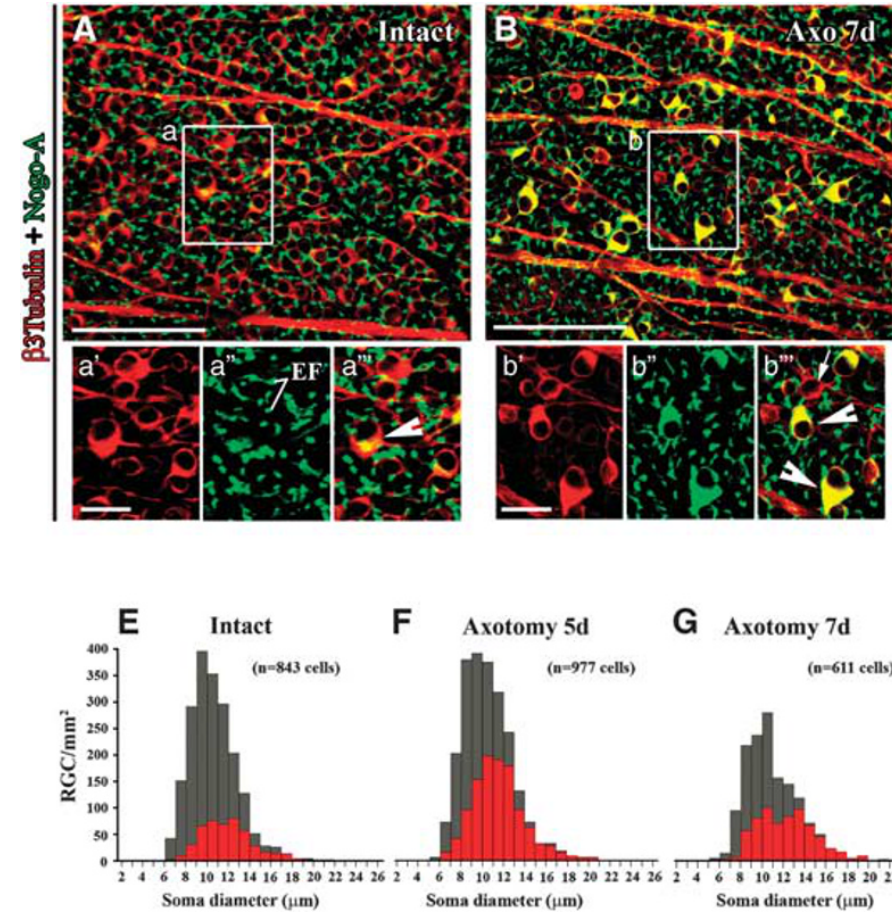

Soma diameter $(\mu \mathrm{m})$

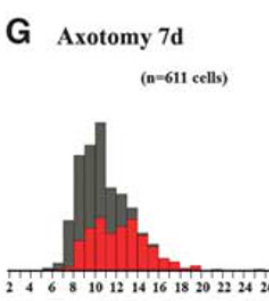

Soma diameter $(\mu \mathrm{m})$

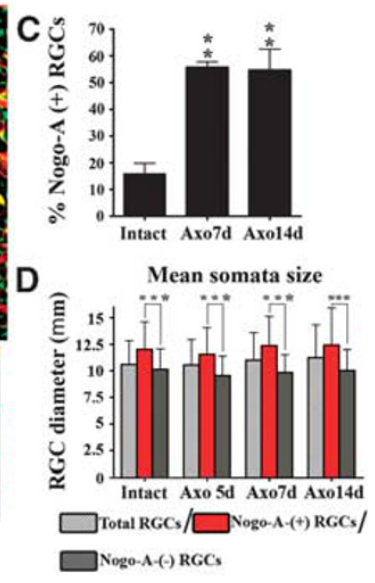

H Axotomy 14d

Figure 2 Nogo-A upregulation in injured retinal ganglion cells. (A) On intact retinal flat-mounts, Nogo-A-positive Müller cell end-feet surrounded RGC cell bodies (a", EF). Only few RGCs exhibited Nogo-A intracellularly $\left(a^{\prime}-a^{\prime \prime \prime}\right)$. (B) Following optic nerve injury, Nogo-A expression dramatically rose in some RGCs, which cell soma appeared bigger than most of the cells, where Nogo-A could not be visualized. (C) Quantitatively, the density of Nogo-A-labelled RGCs significantly increased to $\sim 55 \%$ of the surviving cells at 7 and 14 days post-lesion (ANOVA, ${ }^{* *} P<0.01$ ). (D) The soma diameter of RGCs containing Nogo-A was larger than other RGCs at any time points examined (ANOVA, $\left.{ }^{* \star \star} P<0.001\right)$. $(\mathrm{E}-\mathrm{H})$ The soma size distribution showed that Nogo-A increased in all cell size categories. But, in proportion, cells which somata were bigger than $13 \mu \mathrm{m}$ expressed more Nogo-A than smaller cells. In addition, this sub-population of neurons was better preserved from cell death than the rest of the RGCs. Scale bars: $A, B=100 \mu m ; a^{\prime}, b^{\prime}=25 \mu m$

specificity of the Nogo-A immunostaining was verified on intact and injured Nogo-A KO retinal flat-mount where no signal could be detected (Supplementary Figure S1). Using a Nogo-A/B specific antibody, the general level of Nogo-A and Nogo-B proteins monitored by western blotting were similar in intact and axotomized retinae (Figure 1e, Supplementary Figure S2A-C). When we compared WT and Nogo-A KO retinae by semi-qRT-PCR at 5 days post-injury, the mRNA upregulation of vimentin and GFAP, two indicators of gliosis, did not differ between KO and WT retinae (Figures $1 \mathrm{f}$ and g). This suggests that the bulk part of Nogo-A in the retina is constitutively expressed by the Müller glia and is not influenced by axotomy, in contrast to the cytoskeletal proteins. To obtain a higher resolution of the localization of Nogo-A we analyzed retinal flat-mounts in the layer of the RGCs by confocal microscopy (Figures $2 \mathrm{~A}$ and $\mathrm{B}$ ). In the intact retina, Nogo-A appeared mostly in the end-feet of the Müller cells (EF, Figure 2a- $a^{\prime \prime \prime}$ ) and in a few $\beta 3$ Tubulinlabeled RGC cell bodies (Figure 2a'"', arrowhead). In lesioned retinae, however, Nogo-A dramatically increased in some RGCs (Figure 2 $\mathrm{b}^{\prime \prime \prime}$, arrowheads), whereas other RGCs did not contain Nogo-A at a detectable level (Figure $2 b^{\prime \prime \prime}$, arrow). Quantitatively, Nogo-A was detected in $\sim 15 \%$ of RGCs in the intact retina and rose to $\sim 55 \%$ of the surviving RGCs 7 or 14 days post-lesion (Figure $2 \mathrm{C}$ ). In the superior quadrant, the measurement of the mean soma diameter revealed that Nogo-A-expressing neurons were bigger than RGCs whose cell bodies did not contain Nogo-A (Figure 2D). A large majority of RGCs whose somata exceeded $13 \mu \mathrm{m}$ in diameter expressed Nogo-A after injury (Figures $2 \mathrm{E}-\mathrm{H}$ ). Strikingly, in contrast to Nogo-A, Nogo-B was never observed in intact or injured RGCs (Supplementary Figure S3). The long-lasting upregulation of Nogo-A in injured RGCs points to a particular role for this RTN isoform in the neuronal response to injury.

The ER stress response and apoptotic cell death occur concomitantly with Nogo-A upregulation but not in the same cells after axonal injury. So far, the ER stress activation was not studied after axonal injury. As Nogo-A belongs to the RTN family, which is especially enriched in the ER, we wondered whether neuronal Nogo-A upregulation could reflect the activation of the ER stress response. By semi-qRT-PCR, the pro-apoptotic transcription factors C/EBP homologous protein (CHOP)/GADD153 and c-Jun increased as early as 1 day and peaked at 3 days postaxotomy (Figure 3a). The increase of CHOP/GADD153 protein was confirmed at 3 and 5 days post-lesion by western blotting (Figure 3c, Supplementary Figure S2D). Upstream of CHOP, the active phosphorylated-elF2 $\alpha$ protein was detected in RGCs 3 days after axotomy (Figure $3 b$ ), suggesting that the elF2 $\alpha / \mathrm{CHOP}$ pathway is stimulated by the optic nerve lesion. Accordingly, on retinal sections, the CHOP/GADD153 protein was strongly increased in the 
a

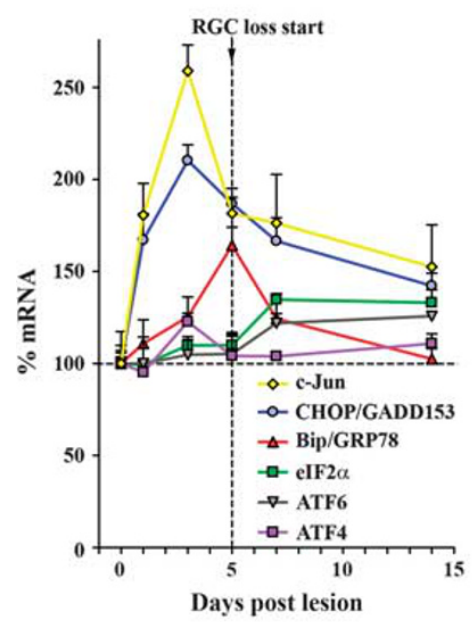

b
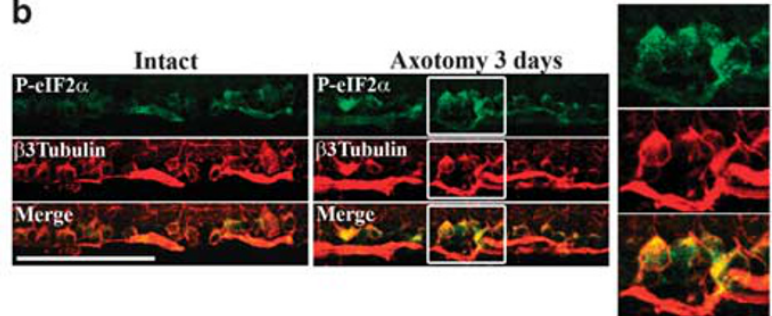

C



d





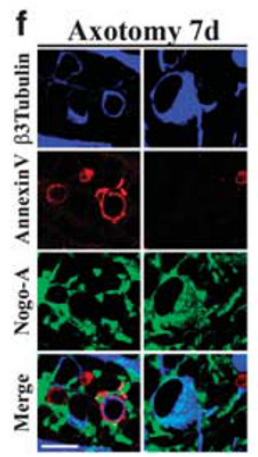

Figure 3 The detection of the ER stress marker CHOP, Nogo-A and annexin $\mathrm{V}$ in the axotomized retina. (a) The time-course of ER stress protein expression was established by semi-qRT-PCR after optic nerve lesion in WT retinae. The pro-apoptotic transcription factor CHOP/GADD153 and c-Jun increased as early as 1 day and peaked at 3 days post-lesion. Bip significantly increased in injured retinae at 5 days relative to intact lysates. (b) By immunohistochemistry, the activated, phosphorylated form of elF2 $\alpha$ appeared more intense in RGCs after axotomy (see 2-fold magnified images on the right). (c) At the protein level, the elevation of CHOP/GADD153 was confirmed in lesioned animals by western blotting. (d) The immunofluorescent signal of CHOP/GADD153 on retinal crossections demonstrated that only axotomized RGCs upregulated this transcription factor. (e) On retinal flat-mounts, large soma-sized RGCs exhibiting a strong signal for Nogo-A were weakly or not positive for CHOP/GADD153. (f) Two hours before perfusion, axotomized WT animals were intraocularly injected with annexin $\mathrm{V}$ to label apoptotic neurons. Fixed retinal flat-mounts were then stained for $\beta 3 T$ Tubulin and Nogo-A. On confocal optical sections, annexin V labelled the cytoplasmic membrane of a few dying RGCs. Large soma-sized RGCs expressing the most Nogo-A were not labelled with annexin V. Scale bars: $B, E=100 \mu \mathrm{m} ; \mathrm{D}=50 \mu \mathrm{m}$, inset $=25 \mu \mathrm{m} ; \mathrm{F}=10 \mu \mathrm{m}$

nucleus of injured RGCs and was not present in other retinal cell layers (Figure 3d). Among the different members of the activating transcription factor (ATF) family, only ATF3 was found to be upregulated in agreement with a previous study (Figures $3 a$ and $6 \mathrm{~g})^{22}$

The relationship between neuronal Nogo-A upregulation, ER stress and neuronal cell death was then analyzed by double immunofluorescence stainings for Nogo-A and CHOP/ GADD153 or the apoptosis marker annexin V, respectively (Figures $3 e$ and f). On 5-day post-axotomy retinal flat-mounts, the large soma-sized RGCs labelled for Nogo-A exhibited a weak or no signal for CHOP/GADD153 (Figure 3e). As the contribution of CHOP/GADD153 to the process of RGC apoptosis is not known, we intraocularly injected annexin $\mathrm{V}$, a protein binding to the cell membrane in the early stage of apoptosis. ${ }^{23}$ For all retinal quadrants, Nogo-A could not be detected in the annexin $\mathrm{V}$-positive neurons, showing that the injury-induced Nogo-A increase is not correlated with cell death (Figure 3f). Nevertheless, it remains possible that in small RGC cells, where Nogo-A was much weaker than in large sized RGCs, Nogo-A was downregulated right before apoptosis and therefore was below the level of detection by immunohistochemistry.

\section{Overexpression but not endogenous Nogo-A} up-regulation influences RGC cell loss after optic nerve lesion. To directly address the influence of Nogo-A on the ER stress activation and RGC apoptosis, Nogo-A was down- or upregulated using AAV2 containing a short hairpin RNA (shRNA) or the gene sequence for Nogo-A, respectively (Figure 4). AAV2 preferentially infects neurons and showed a high selectivity for RGCs in the retina. ${ }^{24}$ By semi-qRT-PCR 


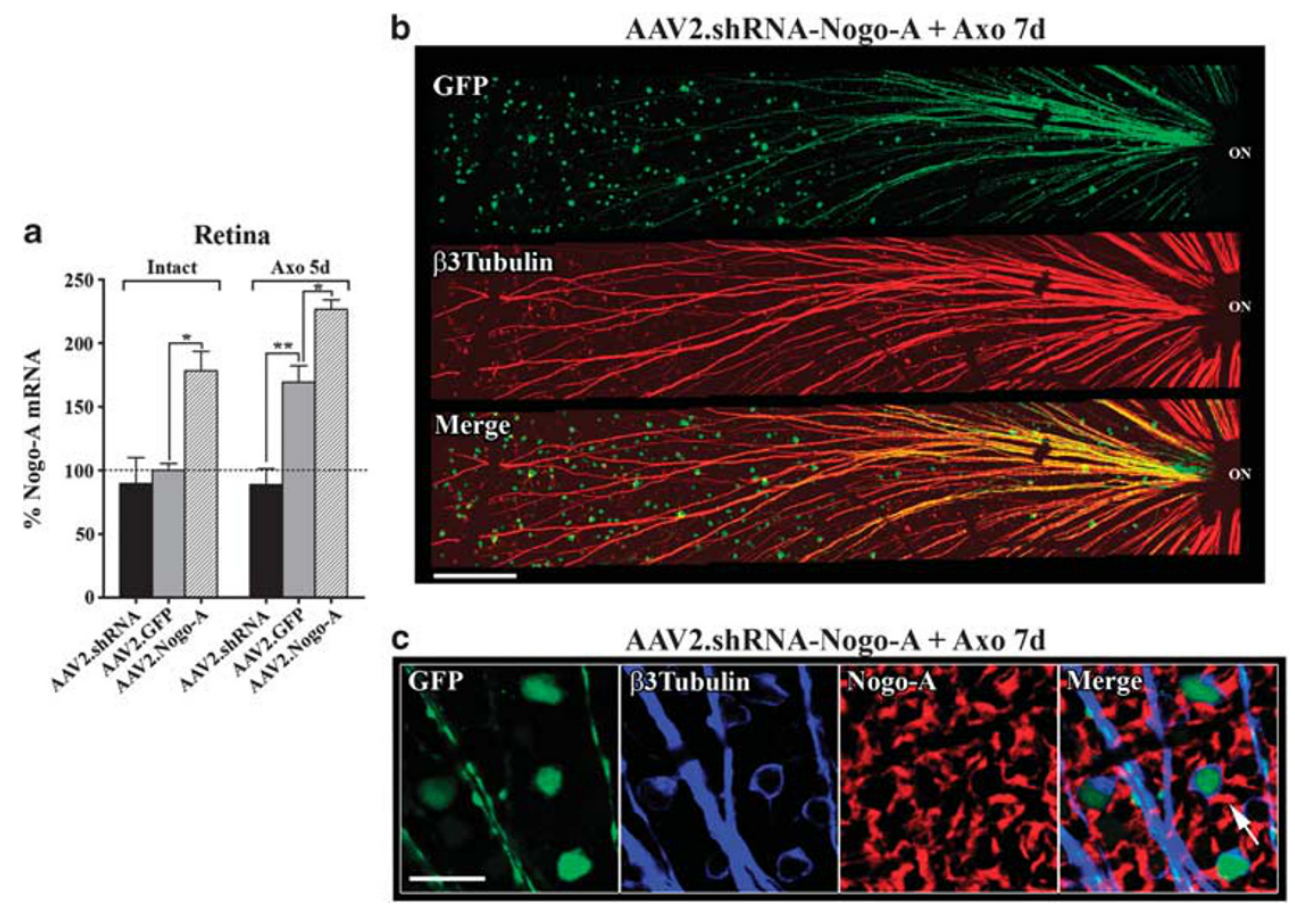

Figure 4 Modulation of neuronal Nogo-A with adeno-associated virus. The modulation of Nogo-A expression by adeno-associated virus vectors (AAV) was validated by semi-qRT-PCR and immunohistochemistry. (a) The semi-qRT-PCR measurement revealed that Nogo-A was efficiently upregulated or downregulated in RGCs after the administration of AAV2.Nogo-A and AAV2.shRNA-Nogo-A, respectively. (b) In vivo, AAV2.shRNA-Nogo-A was delivered intraocularly 4 weeks before optic nerve injury. A large number of RGCs was transfected by AAV2.shRNA-Nogo-A as demonstrated by the co-localization of the RGC-specific marker $\beta 3$ Tubulin and the GFP reporter protein on injured retinal flat-mounts (ON= optic nerve). (c) By immunohistochemistry on retinal flat-mounts, infected RGCs containing the GFP protein and the $\beta 3 T$ Tubulin no longer expressed intracellular Nogo-A after injury although Nogo-A persisted in surrounding Müller cell end-feet (arrow). Scale bars: $B=200 \mu \mathrm{m} ; \mathrm{C}=25 \mu \mathrm{m}$

analysis and immunohistochemistry, AAV2.shRNA-Nogo-A completely abolished injury-induced Nogo-A expression in RGCs, whereas AAV2.Nogo-A was efficient at enhancing the expression of Nogo-A in intact and injured retinae (Figures 4a-c). AAV2.shRNA-Nogo-A infected RGCs before and after optic nerve cut as illustrated by the GFP reporter protein detection on retinal flat-mounts (Figure 4b). Of note, the level of Nogo-A was not decreased in Müller cell end-feet (Figure 4c, arrow).

Two weeks after optic nerve transection, the density of surviving neurons as evaluated by staining RGCs for $\beta 3$ Tubulin on retinal flat-mounts was decreased by $75 \%$ (Figure 5) compared with intact retinae (Supplementary Figure $\mathrm{S} 4 \mathrm{C}$ ). In the whole retina, the average density of surviving RGCs was not significantly changed neither by Nogo-A knock down nor by Nogo-A overexpression compared with the group receiving AAV2.GFP or the untreated animals (Figure 5a). The intraocular injection of AAV2 viruses in the superior retinal quadrant could produce a higher rate of infection in this region possibly leading to stronger effects on survival. ${ }^{24}$ The density of surviving RGCs was therefore separately examined in this part of the retina. Interestingly, in the superior quadrant of the retina, animals infected with AAV2.Nogo-A presented a significantly higher axotomy-induced loss of RGCs at 14 days than any other group (Figure $5 b, D$; $t$-test; ${ }^{*} P<0.05$ ). The death enhancing effect of AAV2.Nogo-A was mainly present in cells with soma sizes smaller than $13 \mu \mathrm{m}$ (Figure 5c, Two-way ANOVA, ${ }^{\star \star} P<0.01$; ${ }^{\star \star \star} P<0.001$ ).
This suggests that the ectopic expression of Nogo-A may exacerbate cell death in cells that have a low endogenous expression of Nogo-A, whereas the majority of large cells upregulating Nogo-A after injury seemed unaffected.

The silencing of Nogo-A with AAV2.shRNA-Nogo-A did not significantly change the CHOP/GADD153 mRNA levels post-lesion when compared with AAV2.GFP-treated retinae (Supplementary Figure S4A). A slight elevation of $\mathrm{CHOP} /$ GADD153 mRNA was, however, noticed in the group treated with AAV2.Nogo-A (Supplementary Figure S4A). In addition, the mRNA level of CHOP/GADD153 was not differentially upregulated in Nogo-A KO and WT retinae 5 days after injury (Supplementary Figure S4B). Consistent with that, the number of RGCs was not much different between WT and Nogo-A KO retinae (Supplementary Figure S4C). These results indicate that axotomy-induced Nogo-A upregulation occurs concomitantly but independently of ER stress activation after optic nerve transection.

Neuronal Nogo-A contributes to the axonal growth response in RGCs. Axonal regeneration was examined 2 weeks after optic nerve crush and neuronal Nogo-A silencing with AAV2.shRNA-Nogo-A. A limited number of axonal fibers was consistently observed in the distal part of the injured optic nerves in absence of treatment or after AAV2.GFP or AAV2.shRNA-luciferase injections (Figures $6 a$ and b). In those respective control nerves, a mean of $58.8 \pm 5.7$ axons or $58.1 \pm 12$ axons per optic nerve were counted at $100 \mu \mathrm{m}$ 
a

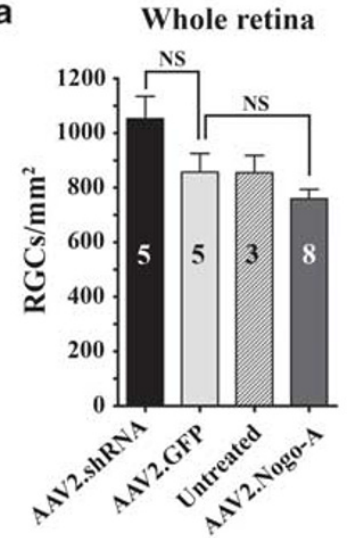

b Superior quadrant

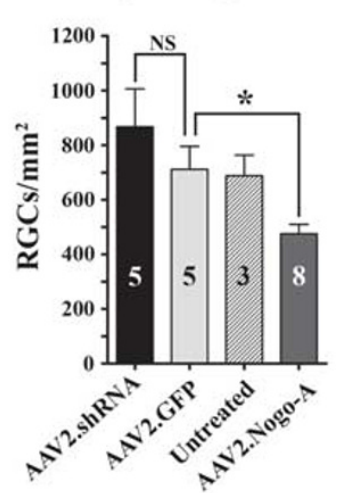

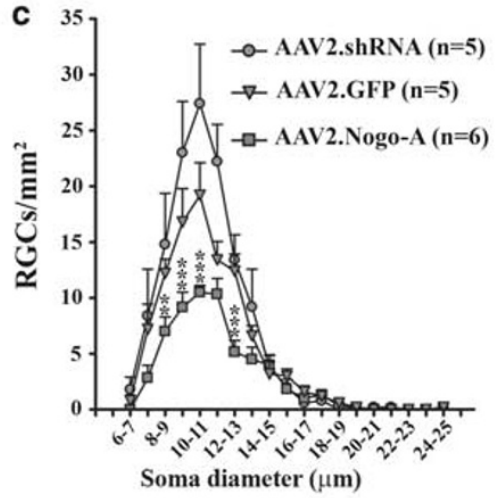

d Axotomy 14 days

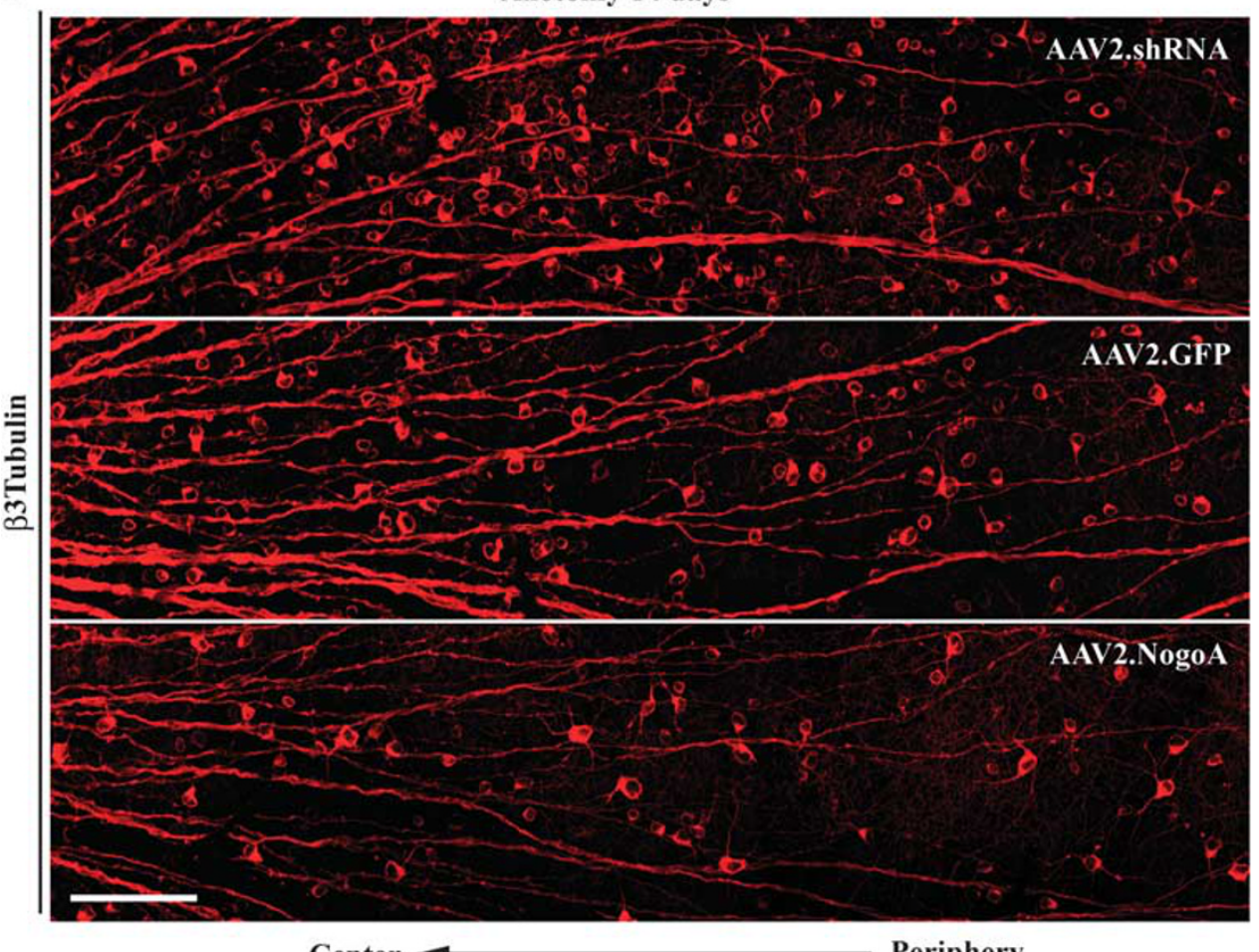

Center

Periphery

Figure 5 The effect of Nogo-A upregulation blockade on RGC survival. (a) In the whole retina, AAV2.shRNA and AAV2.Nogo-A caused no significant (NS) change in the density of surviving RGCs 2 weeks after optic nerve axotomy relative to AAV2.GFP. (b-d) In the superior quadrant of the damaged retina, AAV2.Nogo-A caused more RGC cell loss than AAV2.GFP ( $t$-test, $\left.{ }^{*} P<0.05\right)$. (c) In this region of the retina, the analysis of the soma diameter distribution revealed that the higher loss of RGCs caused by AAV2.Nogo-A occurred for cells smaller than $13 \mu \mathrm{m}$ (Two-way ANOVA, ${ }^{\star \star} P<0.01 ;{ }^{* \star *} P<0.001$ ). Scale bar: $\mathrm{D}=100 \mu \mathrm{m}$

past the lesion site (Figure 6d). At the same distance, animals injected with AAV2.shRNA-Nogo-A showed much less axonal sprouting across the lesion site $(4.4 \pm 2.5$ axons/ optic nerve) (Figures 6a-d). The injury-induced growth response of RGCs was further analyzed by following gene expression changes of Sprr1A, GAP-43 and ATF3, three important regulators of axonal regeneration in the CNS and the PNS. ${ }^{25,26}$ The three growth marker mRNAs increased quickly after injury and peaked at 3-5 days post-optic nerve transection (data not shown). Silencing neuronal Nogo- $A$ in injured retina with AAV2.shRNA-Nogo-A lowered the induction of Sprr1A, GAP-43, and ATF3
mRNA (Figures 6e-g; analysis of variance (ANOVA); $\left.{ }^{*} P<0.05 ;{ }^{* *} P<0.01\right)$. Over-expressing Nogo- $A$ by the administration of AAV2.Nogo-A did not disturb the injuryinduced increase of the three growth markers used (data not shown). These data indicate that neuronal Nogo-A upregulation consecutive to axotomy seems to positively influence or contribute to the neuronal growth response.

Neuronal Nogo-A deletion in Nogo-A KO mice alters RGC responsiveness to growth stimulation. We then investigated how the complete deletion of glial and neuronal Nogo- $A$ in Nogo-A KO mice affected axonal regeneration. 


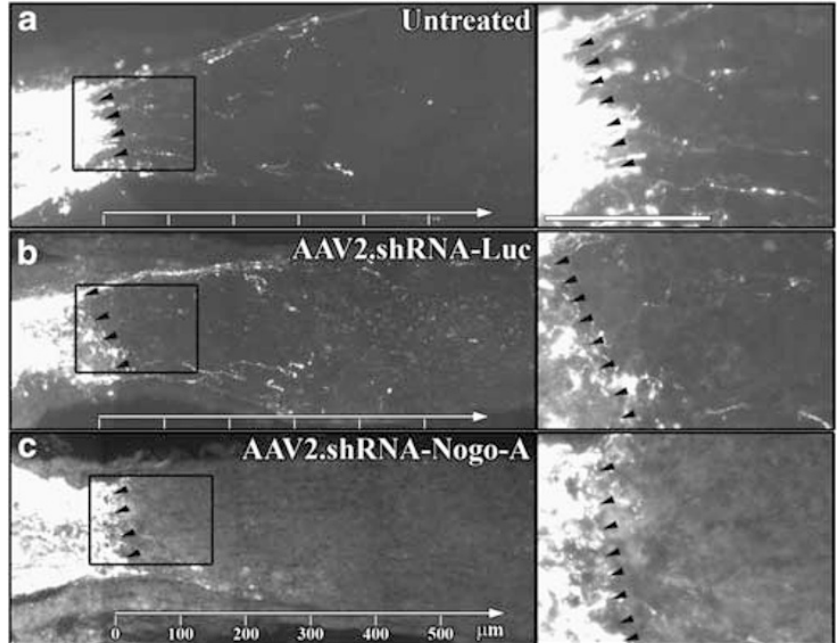

d

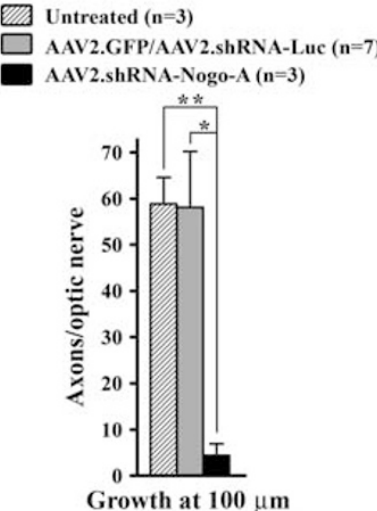

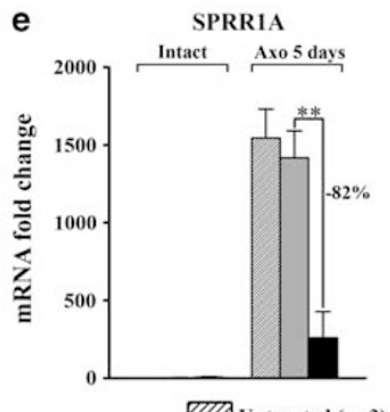

f

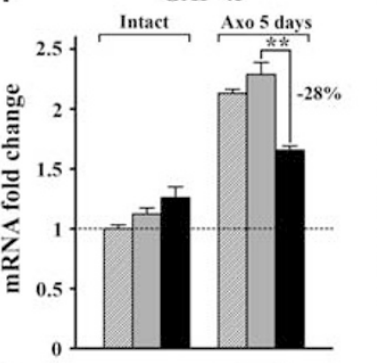

g

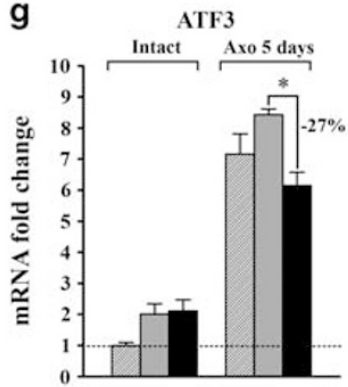

VIAntreated $(\mathrm{n}=3)$

AAV 2.GFP $(n=3)$

AAV 2.shRNA-Nogo-A ( $n=3)$

Figure 6 Silencing Nogo-A in RGCs reduces the neuronal growth response and axonal sprouting in the optic nerve. To study axonal regeneration in the crushed optic nerve, AAV2 vectors were injected 4 weeks before injury and growing axons were traced by injecting intraocularly the cholera toxin- $\beta$ subunit coupled to alexa 594 (CTb-594) the day preceding the killing. Axonal regeneration was analyzed on longitudinal sections at $100 \mu \mathrm{m}$ past the lesion site. (a and $\mathbf{b}$ ) Two weeks after injury, a small number of axons had crossed the lesion site (dark arrowheads) in the optic nerves of untreated mice and of those receiving the control viruses AAV2.GFP or AAV2.shRNA-Luciferase. (c) Almost no axons were able to extend in the distal optic nerve of mice treated with AAV2.shRNA-Nogo-A (right-hand side). (d) Quantitatively, the number of axonal fibers growing across the lesion site was statistically lower after AAV2.shRNA-Nogo-A injection than in groups treated with AAV2.GFP, AAV2.shRNA-luciferase or uninjected (mean \pm S.E.M., ANOVA, Dunnett's post hoc test, ${ }^{*} P<0.05$, $\left.{ }^{\star}{ }^{*} P<0.01\right)$. (e-g) The mRNAs of growth-associated protein Sprr1A, GAP-43 and ATF3 were decreased by AAV2.shRNA-Nogo-A compared with mice administered with AAV2.GFP or those let untreated (ANOVA, ${ }^{\star} P<0.05,{ }^{\star \star} P<0.01$ ). Note that the overexpression of Nogo-A with AAV2.Nogo-A had no effects on the expression of growth-related proteins (data not shown). Scale bar: A inset $=100 \mu \mathrm{m}$

Two weeks after injury, the number of axons growing at 100 , 250, 500 and 1000 microns past the lesion site in Nogo-A KO mice appeared very similar to that of WT mice (Figure 7A). This could mean that the systemic deletion of Nogo-A is not sufficient to promote axonal outgrowth. To place the injured RGCs in an enhanced growth mode, we injected the inflammatory agent Pam3Cys in the vitreous body of lesioned animals. ${ }^{9}$ Injecting Pam3Cys in the vitreous space of C57BL/6 mice strongly activated axonal regrowth in the optic nerve (Figures 7A and C). In Nogo-A KO optic nerves, however, the density of growing axons tended to be lower than in WT animals (Figures 7A, C and D). To evaluate the contribution of neuronal Nogo-A to the axonal growth observed in Nogo-A KO animals, AAV2.Nogo-A was injected 4 weeks before lesion. The growth stimulation with Pam3Cys in this group induced many more growing axons than in Nogo-A KO mice (Figures 7B, D and E). In contrast, the control virus AAV2.GFP did not influence the number of regenerating fibers in WT individuals treated with Pam3Cys (data not shown). Thus, the forced expression of Nogo-A in
RGC neurons can modulate the axonal growth triggered by intraocular inflammation.

Interestingly, the transcript levels of the Nogo-A receptor components $\mathrm{NgR} 1$ and Lingo1 were downregulated by the optic nerve lesion, suggesting that RGCs may be desensitized to myelin inhibitors after injury (Supplementary Figures S5A-B). However, expression changes after lesion did not differ between Nogo-A KO and WT animals. In addition, the gene expression of the pro-apoptotic cytokine $T N F \alpha$ and of survival factors FGF2, CNTF, LIF and BDNF were similarly upregulated in the axotomized retinae of Nogo-A KO and WT mice (Supplementary Figures S5C-D).

Nogo-A levels influence neurite growth in isolated neuronal cells. To assess whether the effects of neuronal Nogo-A on neurite outgrowth were cell-autonomous, Nogo-A was modulated with AAV in the dorsal root ganglia-derived neuronal cell line F11 cultured at single cell density. The levels of Nogo-A were observed by immunocytochemistry in single cells after $A A V$ treatment and forskolin-induced neurite 



Figure 7 Axonal regeneration in Nogo-A KO mice treated with Pam3Cys. Regenerating axons were visualized and quantified in WT and Nogo-A KO mice 2 weeks after micro-crush lesion and axonal tracing with CTb-594 as previously described in Figure 6. (A) The ablation of Nogo-A gene in Nogo-A KO optic nerves failed to promote more axonal outgrowth compared with WT controls. (A, C and D) To switch RGCs into a growth state, the toll-like receptor 2 agonist Pam3Cys ( $5 \mu \mathrm{g})$ was injected the same day as the optic nerve crush and 7 days later in some WT and Nogo-A KO eyes. Intraocular inflammation induced by Pam3Cys dramatically increased the number of CTb-594-labelled fibers in WT optic nerves but tended to be less efficient in Nogo-A KO mice. (B and E) The injection of AAV2.Nogo-A combined to Pam3Cys administration significantly enhanced the number of growing axons in Nogo-A KO mice compared with Nogo-A KO animals treated with Pam3Cys alone (ANOVA, Dunnett's post hoc test, $\left.{ }^{*} P<0.05 ;{ }^{* *} P<0.01,{ }^{* *} P<0.001\right)$

outgrowth (Figures $8 \mathrm{a}-\mathrm{c}$ ). The expression of GFP with control virus did not influence the intensity of Nogo-A staining (Figure 8a). Transfecting F11 cells with Nogo-A shRNA strongly repressed Nogo-A expression (Figure 8b). In contrast, the level of Nogo-A was increased in a way that was tightly correlated with the expression of the His-tag reporter after the addition of AAV2.Nogo-A (Figure 8c). AAV2.GFP or AAV8.GFP did not significantly change neurite outgrowth relative to forskolin treatment alone (Figures $8 \mathrm{~d}, \mathrm{~m}$ ). The knock down of Nogo-A with AAV8.shRNA-Nogo-A caused a statistically significant decrease $(27 \%)$ in neurite outgrowth compared with the AAV8.GFP group (Figure $8 \mathrm{~d}-\mathrm{i}$ and $\mathrm{m}$; ANOVA, $\left.{ }^{\star} P<0.05\right)$. Inversely, the upregulation of Nogo-A significantly increased the length of $F 11$ neurites by $47 \%$ (Figures 8j-m; ANOVA, ${ }^{* \star *} P<0.001$ ). Importantly, the immunofluorescent signals of $\beta 3$ Tubulin and GAP-43 proteins were positively correlated with the expression of Nogo-A in F11 cells infected with AAV2.Nogo-A
(Supplementary Figure S6). The direct correlation between Nogo-A expression and neurite extension supports a cellautonomous and positive effect of Nogo-A on neurite outgrowth.

\section{Discussion}

Following injury to the optic nerve of adult mice, Nogo-A was rapidly and selectively upregulated in RGCs. Although a pronounced ER stress response was induced by the optic nerve axotomy, we could not find a correlation with the accumulation of Nogo-A in RGC cell bodies. The experimental overexpression or downregulation of Nogo-A did not change the survival of lesioned RGCs. In contrast, the regenerative growth response and axonal sprouting were diminished in Nogo-A KO animals and in WT mice where neuronal Nogo- $A$ was downregulated by shRNA transfection. 
a

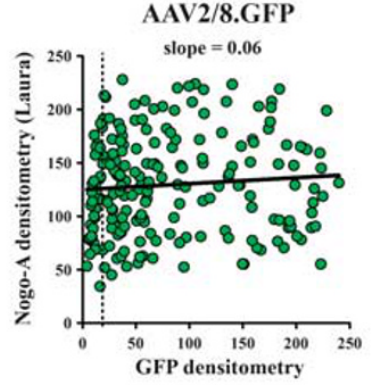

b AAV8.shRNA-Nogo-A

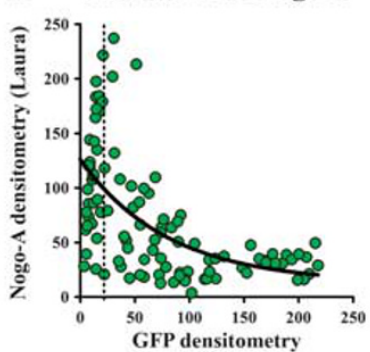

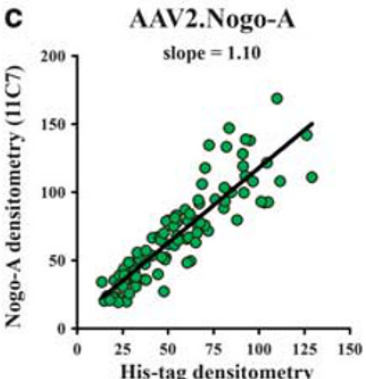
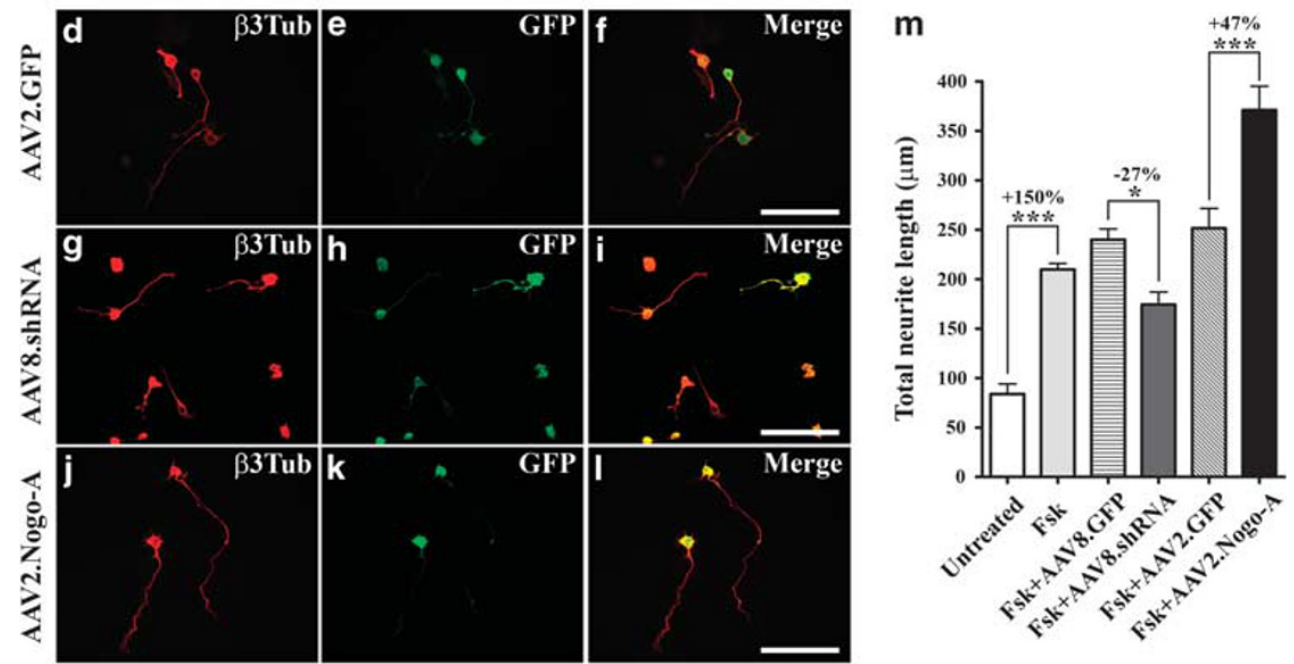

Figure 8 Neurite outgrowth analysis in F11 cells treated with AAV vectors. (a) The densitometric measurement of Nogo-A immunostaining in F11 cells was not affected by AAV8.GFP or AAV2.GFP. The vertical dotted line indicates the threshold under which the GFP intensity was similar to that of untreated cells. AAV8.shRNA-Nogo-A potently blocked Nogo-A expression, (b) whereas the level of Nogo-A increased linearly with the His-tag intensity in AAV2.Nogo-A-treated cells (c). (d-I) The neurites of F11 cells were labelled with an anti- $\beta 3$ Tubulin antibody $48 \mathrm{~h}$ after forskolin-induced differentiation. (m) AAV8.shRNA-Nogo-A reduced the total neurite length of F11 cells, whereas AAV2.Nogo-A had the opposite effect (ANOVA, ${ }^{*} P<0.05,{ }^{* \star *} P<0.001$ ). The mean total neurite length was calculated from 3 independent experiments ( \pm S.E.M.). Scale bar $=200 \mu \mathrm{m}$

Surprisingly, Nogo-A was found in three different cell types in the adult retina and optic nerve: oligodendrocytes, as expected from many other regions of the CNS, a subtype of neuron that is, the RGC after injury, and Müller cells. In the adult retina, the Müller glia was the main cell type producing Nogo-A and Nogo-B. The polarized distribution of Nogo-A and $B$ in the inner Müller end-feet resembled that of RTN 3 or the myelin protein MAG. ${ }^{27,28}$ As for those two other proteins, the physiological role of Nogo-A and $-B$ in the Müller cells is not known. Müller cells react massively to RGC axotomy and have a key role in the inflammatory mechanisms, activating axonal regeneration in the optic nerve after injury, presumably by releasing trophic factors such as CNTF., ${ }^{8,29}$ However, Nogo-A levels remained unchanged after optic nerve lesion in the Müller glia and the loss of Nogo-A in the Müller cells in the $\mathrm{KO}$ mice did not modify the axotomy-induced gliosis.

Although cell surface Nogo-A is well known to exert growth inhibitory effects on neighboring cells via a specific Nogo-A receptor complex, the functions of the high levels of intracellular Nogo-A, in particular in neurons, are largely unknown. As cell death is massive after axotomy in the RGC population of the retina, we studied a possible function of neuronal Nogo-A in RGC apoptosis. Surprisingly, Nogo-A did not contribute at a significant degree to either survival or apoptosis and ER stress. The elevation of CHOP/GADD153 in injured RGCs was not correlated with the increase of Nogo-A, contrary to what was demonstrated for RTN3 in HeLa cells in culture. ${ }^{20}$ Furthermore, neither overexpression nor downregulation of Nogo-A with AAV2 vectors changed significantly the level of CHOP/GADD153 in the retinae. This is consistent with a recent study showing that transfecting COS-7 cells with different RTN proteins did not elicit the activation of CHOP/GADD153 in vitro. ${ }^{15}$ In contrast, RTN1C over-expression in cortical neurons caused the elevation of CHOP/GADD153 and Bip associated with apoptotic death in vivo. ${ }^{19}$ RTN proteins may thus exert opposite effect on the expression of ER stress proteins and the survival of cells. Concerning Nogo-A, the protein was not detectable in apoptotic RGCs labelled with annexin V, and the downregulation of Nogo-A had no global effect on RGC survival. Axotomy-induced Nogo-A upregulation is therefore unlikely to have a major role in neuronal apoptosis in the retina. However, AAV2-mediated Nogo-A downregulation caused less cell death than the overexpression with AAV2.Nogo-A vector, especially in the superior quadrant of the retina. In addition, we did observe that ectopic expression of Nogo-A with AAV2.Nogo-A exacerbated cell death and induced axonal swelling in some neurons, where the infection was 
the highest (data not shown). This probably toxic overexpression is in line with earlier similar observations. ${ }^{18,30}$ The situation in the retina therefore also differs from a model of ALS, where the ablation of the Nogo-A/B gene accelerated the degeneration of ventral motor neuron axons, ${ }^{15}$ whereas the systemic absence of Nogo-A slowed the disease, and Nogo-A overexpression in muscle enhanced neuromuscular endplate degeneration. ${ }^{30}$ Recent studies showed that the neutralization of Lingo or NgR1, 2 receptor components for Nogo-A, promoted RGC survival after optic nerve transection. Therefore, we cannot exclude that the Nogo receptor complex mediates some of the death promoting effects observed after increasing Nogo-A in neuronal cells. ${ }^{31,32}$

Although a majority of RCGs die within 1-2 weeks after optic nerve lesion, the remaining, mostly large diameter neurons show regenerative sprouting and upregulation of growth markers. ${ }^{33,34}$ The increase of Nogo-A especially in these large cells may have a role in the neurite regeneration of RGCs. Nogo-A may be required, although it was not sufficient to confer a higher intrinsic growth capacity to this subset of neurons. Thus, optic axon sprouting was reduced and the lesion-induced increase of Sprr1A, GAP-43 and ATF3 mRNAs was attenuated after neuronal Nogo- $A$ silencing. In turn, the forced expression of Nogo-A in RGCs rescued the decreased axonal regeneration in Nogo-A KO optic nerves treated with Pam3Cys. In isolated F11 cells, the expression of Nogo-A clearly influenced the neuritic length in a positive fashion, suggesting a cell-autonomous effect of Nogo-A on neuronal growth. The exact mechanism is not clear but one may speculate that ER-associated Nogo-A contributes to maintain the neuronal homeostasis by preserving ER structure, protein synthesis and intracellular transport, all processes required for axonal growth and regeneration.

In the present study, Nogo-A KO optic nerve axons did not regenerate better than WT nerves. This result is in contrast with the weaker neurite sprouting that we observed after acute blockade of Nogo-A upregulation with AAV2.shRNA treatment and could be explained by compensatory mechanisms occurring in Nogo-A KO animals. However, earlier studies showed increased axonal regeneration in the optic nerve after blocking Nogo-A with the neutralizing antibody IN-1 secreted by hybridoma cells combined with FGF2 or CNTF. ${ }^{6,35}$ These results are in line with the regeneration enhancement found for corticospinal axons in Nogo-A KO mice after spinal cord lesion and indicate that the inactivation of Nogo-A at the cell surface is beneficial for optic axon growth in vivo. ${ }^{3}$ In Nogo-A $\mathrm{KO}$ mice, the better responsiveness in the corticospinal tract could be because of differences in the intrinsic growth ability between RGCs and cortical neurons or to the severity of the lesion. For example, RGCs die massively after optic nerve crush, whereas spinal cord injury causes a slow shrinkage of the cortical neurons without significant cell death. ${ }^{36}$ Administration of Nogo-A blocking antibodies appears, therefore, as a good strategy to relieve the neurite growth inhibition caused by oligodendrocyte/myelin Nogo-A, without affecting intracellular neuronal Nogo-A.

In summary, our results show for the first time that, in vivo, Nogo-A upregulation in neurons does not take part to ER stress-associated apoptosis, but positively influences the growth response after axonal injury.

\section{Materials and Methods}

Animals. All surgeries were performed on 2-4 month old male C57BL/6 mice. Animal experiments were performed in accordance with the guidelines of the Veterinary Office of the Canton of Zurich. As previously described, Nogo-A KO mice were generated by homologous recombination of exons 2 and 3 in Nogo-A gene in a C57BL/6 background. ${ }^{2}$ The transgenic strain was routinely genotyped by PCRs using the following primer sequences: $5^{\prime}$-AGTGAGTACCCAGCTGCAC- $3^{\prime} ; 5^{\prime}$-CCT ACCCGGTAGAATATCGATAAGC-3'; 5'-TGCTTTGAATTATTCCAAGTAGTCC-3'.

AAV vector production. The recombinant $P A A V-E G F P$ construct was generated by inserting the enhanced green fluorescent protein CDNA into the Xhol and Bgll restriction sites in pAAV-MCS (Stratagene, La Jolla, CA, USA). For pAAV-shNogo856, three different siRNA sequences targeted against the amino portion of Nogo-A were screened for efficiency in vitro. Antisense-loop-sense oligonucleotides corresponding to nucleotides $856-874$ of rat Nogo-A CDNA sequence (shNogo856) were synthesized, annealed and subcloned into the $B b s 1$ and $X b a 1$ sites of the mU6pro plasmid as described. ${ }^{37}$ The U6-shNogo856 fragment was then inserted into the pAAV-EGFP plasmid at the Mlul site upstream of the CMV promoter. The control hairpin siRNA, shLuc, targeted against photinus pyralis luciferase, was constructed by annealing and inserting the described oligonucleotides into the BamH1/EcoR1 sites on the pZac2.1-U6-luc-CMVZsgreen. The pAAV2.Nogo-A construct was generated by inserting the full length of the rat Nogo-A gene sequence between the BamH1 and SnaB1 sites in the pAAVcmv(0.3)nlsLacZ plasmid. All plasmids were verified by sequencing and purified by Maxiprep (Qiagen, Hilden, Germany).

Adeno-associated viral vectors were produced by standard methods. Briefly, triple plasmid co-transfection method ${ }^{38}$ was used with the plasmids described above. After purification by an iodixanol step gradient, the fraction containing the virus was either loaded onto a Hi-Trap Q-HP $1 \mathrm{ml}$ heparin column (GE Healthcare, Chalfont St. Giles, UK) for column purification or directly concentrated and desalted on $100 \mathrm{~K}$ concentrators with PBS as the diluent. Vectors were then titered for DNase-resistant vector genomes by Real-Time qPCR relative to standards or by dot blot assay.

Vector concentrations were calculated in genomes/ $\mathrm{ml}$ with AAV8.GFP at $1.3 \times 10^{13} \mathrm{vg} / \mathrm{ml}$, AAV8.shRNA-Nogo-A at $1.3 \times 10^{13} \mathrm{vg} / \mathrm{ml}$, AAV2.GFP at $2.8 \times 10^{13} \mathrm{vg} / \mathrm{ml}$, AAV2.shRNA-Nogo-A at $2.6 \times 10^{13} \mathrm{vg} / \mathrm{ml}$, AAV2.shRNA-Luciferase at $2.4 \times 10^{13} \mathrm{vg} / \mathrm{ml}$ and AAV2.Nogo-A at $5.1 \times 10^{11} \mathrm{vg} / \mathrm{ml}$. The quantification of GFP positive cells allowed to determine that $\sim 71 \%$ of RGCs were infected by AAV2.shRNA-Nogo-A. By counting the number of His-tag-labeled RGCs, the rate of cells infected by AAV2.Nogo-A represented $\sim 40 \%$. In vitro, the addition of AAV8.shRNA-Nogo-A diminished selectively the Nogo-A protein level in the RGC-5 cell line without affecting Nogo-B, thereby demonstrating the specificity of the construct for Nogo-A. Neither AAV2.Nogo-A nor AAV2.shRNA-Nogo-A affected the number of RGCs in intact retinae; the viruses were therefore not toxic by themselves.

Neuronal survival. The survival of RGCs was assessed after intraorbital optic nerve axotomy. Under general anesthesia, the optic nerve was exposed at $\sim 0.5 \mathrm{~mm}$ from the back of the eye without damaging the ophthalmic artery. In all, 2 weeks after injury the animals were intracardially perfused with $4 \%$ paraformaldehyde (PFA). The retinae were flat-mounted on a glass slide and placed onto a small piece of Whatman filter paper for an overnight post-fixation in $4 \%$ PFA at $4{ }^{\circ} \mathrm{C}$. The RGCs were visualized by immunostaining for $\beta 3$ Tubulin on retinal flat-mounts. The retinae were intensively washed with PBS and incubated with the primary antibody in a solution of PBS containing $0.3 \%$ of Triton-X-100, $5 \%$ of normal serum and $0.05 \%$ sodium azide for $10-14$ days at $4{ }^{\circ} \mathrm{C}$. Then, after washings the retinae were incubated for 3 days with a goat anti-mouse secondary antibody coupled to alexa 594 or $\mathrm{Cy} 3$ at $4{ }^{\circ} \mathrm{C}$. The $\beta 3$ Tubulin-positive RGCs were imaged in the four quadrants of the retina using a Spectral Confocal Microscope TCS SP2 AOBS (Leica, Heerbrugg, Switzerland) with a $\times 40$ oil immersion objective (HCX PL APO $1.25-0.75$ oil CS). Image stacks were acquired in the ganglion cell layer with a step size of $0.5 \mu \mathrm{m}$ and a resolution of $1024 \times 1024$ pixels $(0.37 \mu \mathrm{m} / \mathrm{pixel})$. The number of RGC cell bodies was quantified in grids of $62500 \mu \mathrm{m}^{2}$ at $1 \mathrm{~mm}$ and $1.5 \mathrm{~mm}$ from the optic disk. The density of surviving RGCs was represented in the individual quadrants or in the whole retina per $\mathrm{mm}^{2}$.

Staining of apoptotic neurons with Annexin V. To detect apoptotic neurons, $1.5 \mu \mathrm{l}$. of Annexin $\mathrm{V}$ conjugated to Cy3 (Apoptosis Detection Kit plus, BioVision Incorporated, Lausen, Switzerland, no. K202-100) was injected in the 
eye of axotomized animals, $2 \mathrm{~h}$ before perfusion. After fixation and immunostaining for Nogo-A and $\beta 3$ Tubulin, Annexin V-Cy3-positive cells were visualized on retinal flat-mounts at the peak of apoptosis, 7 days after injury. ${ }^{23}$ Labelled cells were observed by confocal microscopy (see above)

Axonal regeneration analysis. To study axonal regeneration, the optic nerve was crushed intraorbitally. The optic nerve was fully constricted for $15 \mathrm{~s}$ by tying a knot with a 9-0 suture. The suture was then carefully removed and a fundus examination allowed to control the integrity of the ophthalmic artery. Thirteen days after optic nerve crush, the optic axons were anterogradely traced by injecting $1.5 \mu \mathrm{l}$ of $0.5 \%$ cholera toxin $\beta$ subunit conjugated to alexa 594 (CTb-594, Molecular Probes, Basel, Switzerland) into the vitreous space. The day after, the animals were perfused and the optic nerves were processed as described below. CTb-594positive axons were observed on optic nerve longitudinal sections $(14 \mu \mathrm{m})$ with a Zeiss Axioskop 2 Plus microscope (Carl Zeiss, Feldbach, Switzerland) and images were taken with a CCD video camera at $\times 20$. The number of growing axons was estimated at $100 \mu \mathrm{m}, 250 \mu \mathrm{m}, 500 \mu \mathrm{m}$ and $1000 \mu \mathrm{m}$ after the crush site as detailed before. ${ }^{24}$ Optic nerve slices were examined in 3-6 animals per condition. An estimation of the number of axons per optic nerve $(\Sigma)$ was calculated with the following formula: $\Sigma_{d}=\Pi \times R^{2} \times$ (average number of axons $/ \mathrm{mm}$ ) $/ T$. The sum $(\Sigma)$ of axons at a given distance (d) was obtained using the average optic nerve radius $(R)$ of all optic nerves, and a thickness $(T)$ of the tissue slices of $14 \mu \mathrm{m}^{8}$ For statistical analysis, an ANOVA followed by a Bonferroni's or Dunnett's post hoc test was applied for multiple comparisons. Animals presenting ischemia or retinal hemorrhages were excluded from the analysis.

Intraocular injections. AAV viruses, Pam3Cys or the anterograde tracer CTb-594 were injected intraocularly using a $10-\mu$ l Hamilton syringe adapted with a pulled-glass tip and as previously described. ${ }^{24}$ To allow the diffusion of the viruses, the needle was kept in place for 3-4 min and then carefully removed. Attention was paid not to damage the lens or the ciliary bodies that were reported to stimulate growth. 8,39

To up- or downregulate Nogo-A expression in RGCs, 2-4 $\mu$ l of AAV2.Nogo-A or AAV2.shRNA-Nogo-A were injected intraocularly 4-6 weeks before optic nerve injury, a time necessary to obtain an optimal transgene expression in vivo. Similar volumes and concentrations of AAV2.GFP or AAV.shRNA-Luciferase were used as control vectors.

In some groups, axonal regeneration was activated by injecting in the vitreal chamber the toll-like receptor 2 agonist (S)-(2,3-Bis(palmitoyloxy)-(2-RS)-propyl)- $N$ palmitoyl- $(R)$-Cys- $(S)$-Ser-(S)-Lys4-OH (Pam3Cys, EMC Microcolections, Tübingen, Germany). Pam3Cys is a water soluble molecule that was previously shown to induce inflammation-mediated axonal regeneration in the rat optic nerve. ${ }^{9}$ Two injections of Pam3Cys $(2 \mu \mathrm{l}, 2.5 \mu \mathrm{g} / \mu \mathrm{l})$ were performed at the time of the optic nerve lesion and 7 days later.

Retina and optic nerve processing and immunostaining. Adult mice were killed by injecting an overdose of anesthetic intraperitoneally. After intracardiac perfusion with PBS $(0.1 \mathrm{M})$ and $4 \%$ PFA, the eyes were rapidly dissected by removing the cornea and the lens. For retinal cross sections, the eye cups were postfixed in $4 \%$ PFA overnight at $4{ }^{\circ} \mathrm{C}$. The tissues were then cryoprotected in $30 \%$ sucrose overnight and frozen in OCT compound (optimal cutting temperature, Tissue-TEK, Sakura, Alphen aan den Rijn, The Netherlands) with a liquid nitrogen-cooled bath of 2-methylbutane. Optic nerves and retinal sections were cut $(14 \mu \mathrm{m})$ with a cryostat and collected on Superfrost Plus slides (Menzel-Glaser, Braunschweig, Germany). For immunohistochemistry procedure, tissue slices were blocked with $5 \%$ BSA or normal serum, $0.3 \%$ Triton X-100 in PBS for $1 \mathrm{~h}$ at room temperature to avoid unspecific cross-reactivity. Then, primary antibodies were applied in 5\% BSA or normal serum, $0.3 \%$ Triton X-100 in PBS overnight at $4{ }^{\circ} \mathrm{C}$. After PBS washes, sections were incubated with the appropriate secondary antibody for $1 \mathrm{~h}$ at room temperature, and mounted with MOWIOL anti-fading medium (10\% Mowiol 4-88 (w/v) (Calbiochem), in $100 \mathrm{mM}$ Tris, $\mathrm{pH} 8.5$. $25 \%$ glycerol (w/v) and 0.1\% 1,4-diazabicyclo(2.2.2)octane (DABCO)). Primary antibodies were: rabbit anti-Nogo-A (Laura, Rb173A ${ }^{40}$ ) serum $(1: 200)$, rabbit anti-Nogo-A/B (Bianca, $R b 1^{40}$ ) serum $(1: 200)$, rabbit anti-phospho-eukaryotic translation initiation factor $2 \alpha$ (P-elF2 $\alpha, 1: 400$, Cell Signaling, no. 3597), rabbit anti-CHOP/GADD153 (1: 100, Santa Cruz Biotechnology, Heidelberg, Germany, no. sc-575), rabbit anti-glial fibrillary acidic protein (GFAP, 1: 500, Dako, no. 20334), mouse anti-GS $(1: 200-1: 400$, Chemicon, no. MAB302), mouse anti- $\beta 3$ Tubulin ( $1: 1000$, Promega, Madison, WI, USA, no. G712A), mouse anti-Nogo-A
$(11 C 7,401: 200)$. Immunofluorescent labellings were analyzed with a Zeiss Axioskop 2 Plus microscope (Carl Zeiss) and images were taken with a CCD video camera.

Semi-quantitative RT-PCR (qRT-PCR). After cervical dislocation, intact and injured whole retinae (WT, $n=3$ retinae; and Nogo-A KO, $n=4$ retinae) or dorsal hemi-retinae (AAV2-treated, $n=3-4$ retinae) were rapidly dissected in RNA Later solution (Ambion). The samples were placed in eppendorf tubes, flash frozen in liquid nitrogen and stored at $-80^{\circ} \mathrm{C}$ until RNA extraction. The RNeasy RNA isolation kit (Qiagen, Hilden, Germany) was used. Residual genomic DNA was digested by DNase treatment. For reverse transcription, the same amounts of total RNA were transformed by using oligo(dT) primers and M-MLV reverse transcriptase (Promega). The cDNAs corresponding to $10 \mathrm{ng}$ of total RNA were amplified with the following specific primers designed to span intronic sequences or cover exon-intron boundaries: glyceraldehyde-3-phosphate dehydrogenase (GAPDH) (forward, 5' -CAGCAATGCATCCTGCACC-3'; reverse, 5' -TGGACTGTG GTCATGAGCCC-3'), Nogo-A/RTN4a (forward, 5'-CAGTGGATGAGACCCTTTT TG-3'; reverse, 5'-GCTGCTCCTTCAAATCCATAA-3'), c-Jun (forward, 5'-AAAA CCTTGAAAGCGAAAA-3'; reverse, $5^{\prime}$-TAACAGTGGGTGCCAACTCA-3'), CHOP/ GADD153 (forward, 5'-ACACCACCACACCTGAAAGCAG-3'; reverse, 5'-TGACTG GAATCTGGAGAGCGAG-3'), Bip/GRP78 (forward, 5'-TGCAGCAGGACATCAAG TTC-3'; reverse, $5^{\prime}$-CAGCAATAGTGCCAGCATCT-3'), elF2 $\alpha$ (forward, -5'-CCAG GAAGTGACAAGCCATT-3'; reverse, $5^{\prime}$-TCAGGATCACCAGAAGCAGA-3'), ATF3 (forward, 5'-ACCTCCTGGGTCACTGGTATTTG-3'; reverse, 5'-TTCTTTCTCGC CGCCTCCTTTTCC-3'), ATF4 (forward, 5'-ATGGCGTATTAGAGGCAGCAGT-3'; reverse, 5'-TGTTCAGGAAGCTCATCTCGGT-3'), ATF6 (forward, 5'-TGCCTTG GGAGTCAGACCTA-3', reverse, 5'-AGGAAGCCGGAGAAAGAGG-3'), GAP-43 (forward, 5'-TGCTGTCACTGATGCTGCT-3' ${ }^{\prime}$, reverse, $5^{\prime}$-GGCTTCGTCTACAGCG TCTT-3'), small proline-rich protein $1 \mathrm{~A}$ (Sprr1A; forward, $5^{\prime}$-GAACCTGCTCTTCTC TGAGT-3', reverse, 5'-AGCTGAGGAGGTACAGTG-3'), vimentin (forward, 5'-TA CAGGAAGCTGCTGGAAGG-3', reverse, $5^{\prime}$-TGGGTGTCAACCAGAGGAA-3'), GFAP (forward, 5' - CCACCAAACTGGCTGATGTCTAC-3', reverse, 5'-TTCTCTC CAAATCCACACGAGC-3'), NgR/RTN4R (forward, 5'-CTCGACCCCGAAGATGA AG-3'; reverse 5'-TGTAGCACACACAAGCACCAG-3'), LINGO (forward, 5'-AAGT GGCCAGTTCATCAGGT-3'; reverse 5'-TGTAGCAGAGCCTGACAGCA-3'), Tumor Necrosis Factor $\alpha$ (TNF $\alpha$, forward, $5^{\prime}$-CCACGCTCTTCTGTCTACTGA-3'; reverse $5^{\prime}$-GGCCATAGAACTGATGAGAGG-3'), Fibroblast Growth Factor (FGF2, forward 5'-TGTGTCTATCAAGGGAGTGTGTGC-3'; reverse $5^{\prime}$-ACCAACTGGAGTATTT CCGTGACCG-3'), CNTF (forward 5'-CTCTGTAGCCGCTCTATCTG-3'; reverse 5'-GGTACACCATCCACTGAGTC-3'), Leukemia Inhibitory Factor (LIF, forward 5'-AATGCCACCTGTGCCATACG-3'; reverse 5'-CAACTTGGTCTTCTCTGTCC CG-3'), BDNF (forward, 5'-CAAAGCCACAATGTTCCACCAG-3'; reverse 5'-GATG TCGTCGTCAGACCTCTCG-3v).

Gene expression was analyzed by real-time RT-PCR with a polymerase ready mix (Light Cycler480, SYBR Green I Master; Roche Diagnostics, Rotkreutz, Switzerland) and a thermocycler (LightCycler; Roche Diagnostics). The analysis of the melting curve of each amplified PCR product and the visualization of the PCR amplicons on $1.5 \%$ agarose gels allowed to control the specificity of the amplification. For relative quantification of gene expression, mRNA levels were normalized to GAPDH using the comparative threshold cycle $\left(\Delta \Delta^{\mathrm{CT}}\right)$ method. Similar normalized results were obtained by using $\beta$-actin as housekeeping gene. For calibration, a control sample was used to calculate the relative values. Each reaction was done in triplicate.

Western blot analysis. Three mice per group were killed by cerebral dislocation and retinae and optic nerves were quickly dissected out and flash frozen in liquid nitrogen. Tissues were kept at $-80^{\circ} \mathrm{C}$ until extraction in lysis buffer $(20 \mathrm{mM}$ Tris- $\mathrm{HCl}, 0.5 \%$ CHAPS, pH 8 ) containing protease inhibitors (Complete mini, Roche diagnostics). The samples were fully homogenized and let on ice for $60 \mathrm{~min}$. After centrifugation for $15 \mathrm{~min}$ at $15000 \times \mathrm{g}, 4^{\circ} \mathrm{C}$, the supernatant was collected in new eppendorf tubes, and stored at $-80^{\circ} \mathrm{C}$. Proteins ( $20 \mu \mathrm{g} /$ lane) were separated by electrophoresis on a $4-12 \%$ polyacrylamide gel and transferred to nitrocellulose membranes. Blots were pre-incubated in a blocking solution of $2 \%$ Top Block (Lubio Science, Lucerne, Switzerland) in TBST 0.2\% (Tris-base $0.1 \mathrm{M}, 0.2 \%$ Tween-20, $\mathrm{pH} 7.4$ ) for $1 \mathrm{~h}$ at room temperature, incubated with primary antibodies overnight at $4^{\circ} \mathrm{C}$ and after washing, with a horseradish peroxidase-conjugated anti-mouse or anti-rabbit antibody (1:10000-1:25000; Pierce Biotechnology, Lausanne, Switzerland). Primary antibodies were rabbit anti-Nogo-A/B (Bianca, $R b 1^{40}$ ) serum $(1: 20000)$, rabbit anti-CHOP/GADD153 $(1: 100$, Santa Cruz Biotechnology, no. sc-575) and mouse anti-GAPDH (1:10000; Abcam, Cambridge, UK). 
Protein bands were detected by adding SuperSignal West Pico Chemiluminescent Substrate (Pierce Biotechnology) and after exposure of the blot in a Stella detector. The quantification of the band intensity was done with the Image $\mathrm{J}$ software (NIH, Bethesda, MD, USA).

Cell cultures. Freshly dissociated Müller cells were prepared from C57BL/6 adult mouse retinae (3-4 month old). In brief, retinae were rapidly isolated in $\mathrm{CO}_{2}$-independent medium after cervical dislocation. After two washes in Ringer solution, the retinae were incubated for $35 \mathrm{~min}$ at $37^{\circ} \mathrm{C}$ in a digestion solution containing $1.5 \mathrm{mg} / \mathrm{ml}$ of papain and $12.5 \mathrm{mM}$ of L-cystein dissolved in Ringer buffer. The retinae were then gently triturated in a solution of Dulbecco'S modified Eagle's medium (DMEM) with 10\% FBS, $0.1 \mathrm{mg} / \mathrm{ml}$ DNase I using a fire-polished Pasteur pipette. The cell suspension was then centrifuged at $250 \times g$ for $3 \mathrm{~min}$ and washed twice with medium. At the last centrifugation, the cell pellet was re-suspended in $\sim 1 \mathrm{ml}$ of medium and cells were seeded on poly-D-lysine/laminin-coated glass coverslips. For immunofluorescence, $\sim 1 \mathrm{~h}$ after cell adhesion, the cells were fixed with 4\% PFA for $30 \mathrm{~min}$, rinsed three times with PBS and incubated for $1 \mathrm{~h}$ at room temperature in a blocking solution (0.1 M PBS; $0.1 \%$ triton-X100; $5 \%$ normal goat serum). The rabbit anti-Nogo-A antibody Laura $(1: 200)$ and the mouse anti-GS antibody $(1: 400)$ were then added overnight at $4{ }^{\circ} \mathrm{C}$. After washes and secondary antibody incubation the stained cells were imaged with a confocal microscope as described for tissue sections.

The efficacy of AAV.shRNA-Nogo-A to downregulate Nogo-A protein expression was tested on the RGC-5 cell line ${ }^{41}$ (a gift from Dr. Krishnamoorthy, Fort Worth, TX, USA) by western blotting. One $\mu$ l of AAV8.shRNA-Nogo-A or AAV8.GFP control virus $\left(1.3 \times 10^{13} \mathrm{vg} / \mathrm{ml}\right)$ was added to RGC-5 cells plated at a density of $200000 \mathrm{cell} / \mathrm{s} / \mathrm{ml}$ in 6-well plates, in serum-free DMEM. Five days later, RGC-5 cells were lysed with CHAPS buffer and proteins were separated by electrophoresis as described above.

The effects of AAV2.Nogo-A, AAV8.shRNA-Nogo-A, AAV2.GFP and AAV8.GFP on neurite outgrowth were evaluated in vitro by infecting F11 cells. The F11 cell line, kindly provided by Prof. RE van Kesteren (Amsterdam, The Netherlands), was maintained as described before. ${ }^{42} \mathrm{~F} 11$ cells were plated at a density of 100000 cells per well in 6-well plates and were treated with AAV viruses $24 \mathrm{~h}$ later. Four days after infection, F11 cells were transferred to 4-well dishes at a density of 2000 cells per well. Twenty four hours after cell spreading, F11 cells were differentiated by adding $10 \mu \mathrm{M}$ of forskolin for $48 \mathrm{~h}$. To visualize neurite extension, F11 cells were co-immunostained for $\beta 3$ Tubulin and GFP or His-tag to observe the cells infected with AAV2.GFP/AAV8.GFP/AAV8.shRNA-Nogo-A or AAV2.Nogo-A, respectively. In three independent experiments, the measurement of neurite length was carried out using the Neuron $\mathrm{J}$ plugin in the Image $\mathrm{J}$ software $(\mathrm{NIH})$. The mean of the total neurite length was calculated for the different treatments using between 318 and 466 cells which neurite length $\geq 25 \mu \mathrm{m}$. The level of GFP (AAV2.GFP/AAV8.GFP or AAV8.shRNA-Nogo-A), His peptide (AAV2.Nogo-A) and Nogo-A expressions were assessed in $\mathrm{F} 11$ cells bodies by densitometry in Image $\mathrm{J}$.

\section{Conflict of Interest}

The authors declare no conflict of interest.

Acknowledgements. We want to thank Dr. Jody $L$ Martin for the preparation of the AAV.shRNA-Nogo-A and the INSERM viral vector core facility in Nantes, France, for the production of AAV2.Nogo-A and AAV2.GFP. This work was supported by Swiss National Science Foundation (SNF) Grant no. 31-122527/1 and the SNF National Center of Competence in Research 'Neural Plasticity and Repair'.

1. Schwab ME. Nogo and axon regeneration. Curr Opin Neurobiol 2004; 14: 118-124.

2. Simonen M, Pedersen V, Weinmann O, Schnell L, Buss A, Ledermann B et al. Systemic deletion of the myelin-associated outgrowth inhibitor Nogo-A improves regenerative and plastic responses after spinal cord injury. Neuron 2003; 38: 201-211.

3. Dimou L, Schnell L, Montani L, Duncan C, Simonen M, Schneider R et al. Nogo-A-deficient mice reveal strain-dependent differences in axonal regeneration. J Neurosci 2006; 26 : 5591-5603.

4. Fischer D, He Z, Benowitz LI. Counteracting the Nogo receptor enhances optic nerve regeneration if retinal ganglion cells are in an active growth state. J Neurosci 2004; 24 1646-1651.

5. Bartsch U, Bandtlow CE, Schnell L, Bartsch S, Spillmann AA, Rubin BP et al. Lack of evidence that myelin-associated glycoprotein is a major inhibitor of axonal regeneration in the CNS. Neuron 1995; 15: 1375-1381.
6. Weibel D, Cadelli D, Schwab ME. Regeneration of lesioned rat optic nerve fibers is improved after neutralization of myelin-associated neurite growth inhibitors. Brain Res 1994; 642: 259-266.

7. Yin $Y$, Cui $Q, L i$ Y, Irwin N, Fischer D, Harvey AR et al. Macrophage-derived factors stimulate optic nerve regeneration. J Neurosci 2003; 23: 2284-2293.

8. Leon S, Yin Y, Nguyen J, Irwin N, Benowitz LI. Lens injury stimulates axon regeneration in the mature rat optic nerve. J Neurosci 2000; 20: 4615-4626.

9. Hauk TG, Leibinger M, Muller A, Andreadaki A, Knippschild U, Fischer D. Stimulation of axon regeneration in the mature optic nerve by intravitreal application of the toll-like receptor 2 agonist Pam3Cys. Invest Ophthalmol Vis Sci 2009; 51: 459-464.

10. Fischer $\mathrm{D}$, Petkova V, Thanos S, Benowitz LI. Switching mature retinal ganglion cells to a robust growth state in vivo: gene expression and synergy with RhoA inactivation. J Neurosci 2004; 24: 8726-8740.

11. Park KK, Liu K, Hu Y, Smith PD, Wang C, Cai B et al. Promoting axon regeneration in the adult CNS by modulation of the PTEN/mTOR pathway. Science 2008; 322: 963-966.

12. Smith PD, Sun F, Park KK, Cai B, Wang C, Kuwako K et al. SOCS3 deletion promotes optic nerve regeneration in vivo. Neuron 2009; 64: 617-623.

13. Cheatwood JL, Emerick AJ, Schwab ME, Kartje GL. Nogo-A expression after focal ischemic stroke in the adult rat. Stroke 2008; 39: 2091-2098.

14. Marklund N, Fulp CT, Shimizu S, Puri R, McMillan A, Strittmatter SM et al. Selective temporal and regional alterations of Nogo-A and small proline-rich repeat protein $1 \mathrm{~A}$ (SPRR1A) but not Nogo-66 receptor (NgR) occur following traumatic brain injury in the rat. Exp Neurol 2006; 197: 70-83.

15. Yang YS, Harel NY, Strittmatter SM. Reticulon-4A (Nogo-A) redistributes protein disulfide isomerase to protect mice from SOD1-dependent amyotrophic lateral sclerosis. J Neurosci 2009; 29: 13850-13859.

16. Kilic E, Elali A, Kilic U, Guo Z, Ugur M, Uslu U et al. Role of Nogo-A in neuronal survival in the reperfused ischemic brain. J Cereb Blood Flow Metab 2010; 30: 969-984.

17. HuX, Shi Q, Zhou X, He W, Yi H, Yin X et al. Transgenic mice overexpressing reticulon 3 develop neuritic abnormalities. Embo J 2007; 26: 2755-2767; Epub 2007 May 3.

18. Aloy EM, Weinmann O, Pot C, Kasper H, Dodd DA, Rulicke T et al. Synaptic destabilization by neuronal Nogo-A. Brain Cell Biol 2006; 35: 137-157.

19. Fazi B, Biancolella M, Mehdawy B, Corazzari M, Minella D, Blandini $F$ et al. Characterization of gene expression induced by RTN- $1 C$ in human neuroblastoma cells and in mouse brain. Neurobiol Dis 2010; 40: 634-644.

20. Wan Q, Kuang E, Dong W, Zhou S, Xu H, Qi Y et al. Reticulon 3 mediates Bcl-2 accumulation in mitochondria in response to endoplasmic reticulum stress. Apoptosis 2007; 12: 319-328.

21. Di Sano F, Fazi B, Tufi R, Nardacci R, Piacentini M. Reticulon- $1 C$ acts as a molecular switch between endoplasmic reticulum stress and genotoxic cell death pathway in human neuroblastoma cells. J Neurochem 2007; 102: 345-353.

22. Nie DY, Zhou ZH, Ang BT, Teng FY, Xu G, Xiang T et al. Nogo-A at CNS paranodes is a ligand of Caspr: possible regulation of $\mathrm{K}(+)$ channel localization. Embo $\mathrm{J}$ 2003; 22: 5666-5678.

23. Cordeiro MF, Guo L, Luong V, Harding G, Wang W, Jones HE et al. Real-time imaging of single nerve cell apoptosis in retinal neurodegeneration. Proc Natl Acad Sci USA 2004; 101: 13352-13356.

24. Pernet V, Hauswirth WW, Di Polo A. Extracellular signal-regulated kinase 1/2 mediates survival, but not axon regeneration, of adult injured central nervous system neurons in vivo. J Neurochem 2005; 93: 72-83.

25. Seifffers $R$, Mills $C D$, Woolf $C J$. ATF3 increases the intrinsic growth state of DRG neurons to enhance peripheral nerve regeneration. J Neurosci 2007; 27: 7911-7920.

26. Bonilla IE, Tanabe K, Strittmatter SM. Small proline-rich repeat protein $1 \mathrm{~A}$ is expressed by axotomized neurons and promotes axonal outgrowth. J Neurosci 2002; 22: 1303-1315.

27. Kumamaru E, Kuo CH, Fujimoto T, Kohama K, Zeng LH, Taira E et al. Reticulon3 expression in rat optic and olfactory systems. Neurosci Lett 2004; 356: 17-20.

28. Stefansson K, Molnar ML, Marton LS, Molnar GK, Mihovilovic M, Tripathi RC et al. Myelinassociated glycoprotein in human retina. Nature 1984; 307: 548-550.

29. Leibinger M, Muller A, Andreadaki A, Hauk TG, Kirsch M, Fischer D. Neuroprotective and axon growth-promoting effects following inflammatory stimulation on mature retinal ganglion cells in mice depend on ciliary neurotrophic factor and leukemia inhibitory factor. J Neurosci 2009; 29: 14334-14341.

30. Jokic N, Gonzalez de Aguilar JL, Dimou L, Lin S, Fergani A, Ruegg MA et al. The neurite outgrowth inhibitor Nogo-A promotes denervation in an amyotrophic lateral sclerosis model. EMBO Rep 2006; 7: 1162-1167.

31. Fu QL, Hu B, Wu W, Pepinsky RB, Mi S, So KF. Blocking LINGO-1 function promotes retinal ganglion cell survival following ocular hypertension and optic nerve transection. Invest Ophthalmol Vis Sci 2008; 49: 975-985.

32. Fu QL, Liao XX, Li X, Chen D, Shi J, Wen W et al. Soluble Nogo66 receptor prevents synaptic dysfunction and rescues retinal ganglion cell loss in chronic glaucoma. Invest Ophthalmol Vis Sci 2011; 52: 8374-8380

33. Cui Q, Harvey AR. CNTF promotes the regrowth of retinal ganglion cell axons into murine peripheral nerve grafts. Neuroreport 2000; 11: 3999-4002.

34. Watanabe M, Sawai H, Fukuda Y. Number, distribution, and morphology of retinal ganglion cells with axons regenerated into peripheral nerve graft in adult cats. J Neurosci 1993; 13: 2105-2117. 
35. Cui Q, Cho KS, So KF, Yip HK. Synergistic effect of Nogo-neutralizing antibody IN-1 and ciliary neurotrophic factor on axonal regeneration in adult rodent visual systems. J Neurotrauma 2004; 21: 617-625.

36. Wannier T, Schmidlin E, Bloch J, Rouiller EM. A unilateral section of the corticospinal tract at cervical level in primate does not lead to measurable cell loss in motor cortex. J Neurotrauma 2005; 22: 703-717.

37. Yu JY, DeRuiter SL, Turner DL. RNA interference by expression of short-interfering RNAs and hairpin RNAs in mammalian cells. Proc Natl Acad Sci USA 2002; 99: 6047-6052; Epub 2002 Apr 23.

38. Zolotukhin S, Potter M, Hauswirth WW, Guy J, Muzyczka N. A 'humanized' green fluorescent protein cDNA adapted for high-level expression in mammalian cells. $J$ Viro 1996; 70: 4646-4654.
39. Fischer $\mathrm{D}$, Pavlidis $\mathrm{M}$, Thanos $\mathrm{S}$. Cataractogenic lens injury prevents traumatic ganglion cell death and promotes axonal regeneration both in vivo and in culture. Invest Ophthalmol Vis Sci 2000; 41: 3943-3954.

40. Oertle T, van der Haar ME, Bandtlow CE, Robeva A, Burfeind P, Buss A et al. Nogo-A inhibits neurite outgrowth and cell spreading with three discrete regions. J Neurosci 2003; 23: 5393-5406.

41. Krishnamoorthy RR, Agarwal P, Prasanna G, Vopat K, Lambert W, Sheedlo HJ et al. Characterization of a transformed rat retinal ganglion cell line. Brain Res Mol Brain Res 2001; 86: 1-12.

42. MacGillavry HD, Stam FJ, Sassen MM, Kegel L, Hendriks WT, Verhaagen J et al. NFIL3 and cAMP response element-binding protein form a transcriptional feedforward loop that controls neuronal regeneration-associated gene expression. J Neurosci 2009; 29: 15542-15550.

Supplementary Information accompanies the paper on Cell Death and Differentiation website (http://www.nature.com/cdd) 